\title{
Vortioxetine improved social and cognitive deficits in acute ketamine model of schizophrenia in rats
}

\author{
Gökhan ÜNAL 1, 2 * (D), Mehmet TAŞKIRAN 3 (D) \\ 1 Department of Pharmacology, Faculty of Pharmacy, Erciyes University, Kayseri, Turkey. \\ 2 Experimental Research and Application Center (DEKAM), Erciyes University, Kayseri, Turkey \\ 3 Department of Biology, Faculty of Science, Erciyes University, Kayseri, Turkey. \\ * Corresponding Author. E-mail: gokhanunal@erciyes.edu.tr (G.U.); Tel. +90-352-207 6666.
}

Received: 16 July 2020 / Revised: 24 August 2020/ Accepted: 27 August 2020

\begin{abstract}
Schizophrenia is a devastating psychiatric disorder with its complex symptoms and neurobiology. It has been known that serotonergic dysregulation contributes to the pathogenesis of schizophrenia, although dopaminergic and glutamatergic systems are thought to have central roles in neurobiology. Vortioxetine is a new multimodal antidepressant that shows agonistic effects on 5-HT1A and partial agonist on 5-HT1B receptors, antagonistic effects on the serotonergic 5-HT3, and 5-HT7 receptors and high-affinity inhibitory effect on serotonin reuptake pumps. A limited number of studies suggest that vortioxetine may have a potential therapeutical effect on schizophrenia, especially on negative and cognitive symptoms. Herein, we first investigated the potential beneficial effects of vortioxetine on positive, negative, and cognitive symptoms of schizophrenia in the acute ketamine model of rats. For these reasons, Wistar albino rats grouped as saline, ketamine (10 or $15 \mathrm{mg} / \mathrm{kg})$, vortioxetine $(10 \mathrm{mg} / \mathrm{kg})$, and ketamine+vortioxetine $(10 \mathrm{mg} / \mathrm{kg})(\mathrm{n}=8$ in each group). Social interaction (SI), novel object recognition (NOR), and prepulse inhibition (PPI) tests were performed to evaluate schizophrenia-like behaviors in rats. Vortioxetine attenuated the social deficits induced by ketamine in the SI test. In NOR, ketamine reduced the discrimination index and vortioxetine reversed this effect of ketamine in rats. Ketamine decreased the prepulse inhibition (\%) compared to saline, and vortioxetine treatment exacerbated the ketamine reduced prepulse inhibition (\%) in rats. In conclusion, our results suggested that vortioxetine may have beneficial effects, especially on negative symptoms of schizophrenia. It will be beneficial to confirm our study in different schizophrenia models of rodents and investigating the possible underlying mechanism of these effects.
\end{abstract}

KEYWORDS: Vortioxetine; ketamine; schizophrenia; novel object recognition; social interaction.

\section{INTRODUCTION}

Schizophrenia is one of the complex neuropsychiatric diseases with its approximately $1 \%$ prevalence. Its symptomology consists of positive symptoms such as hallucination and delusion; negative symptoms lack motivation and decreased social behaviors and cognitive deficits in attention and memory. The current treatment regimen frequently consists of atypical (second generation) and sometimes, typical (first generation) antipsychotic drugs. Atypical antipsychotics are mechanistically differentiated from the typical ones due to its serotonergic modulation, especially on 5-HT2A receptors and clinically differentiated with a better side effect profile and relative effectiveness on negative and cognitive function [1]. Recent non-sponsored, large scale clinical studies showed that atypical drugs have no clear superiority on typical drugs for the effectiveness of negative and cognitive symptoms of schizophrenia. Therefore, the current antipsychotic drugs have poor effects on negative and cognitive symptoms though they are partially successful in preventing positive symptoms. In addition to the ineffectiveness of these drugs, several serious side effects, including tardive dyskinesia, hyperprolactinemia, metabolic syndrome, and agranulocytosis may happen in the subjects with schizophrenia [2]. Therefore, researches have focused on novel therapeutical approaches for better treatment for schizophrenia.

Glutamatergic hypoactivity is one of the hit hypotheses about the pathophysiology of schizophrenia. It is based on the decrease of glutamatergic N-Methyl-D-Aspartate (NMDA) receptor function in the corticalbrainstem pathway in the brain [3]. Previous studies have demonstrated that the administration of NMDA receptor antagonists such as Ketamine and Phencyclidine reveal psychosis-like statements in healthy volunteers and schizophrenia-like behavioral and neurobiological changes in rodents [4-6]. It has been shown that acute administration of NMDA receptor antagonists is beneficial for scanning the antipsychotic like effects of novel therapeutical approaches in rats [5].

How to cite this article: Ünal G, Taşkıran M. Vortioxetine improved social and cognitive deficits in acute ketamine model of schizophrenia in rats. J Res Pharm. 2020; 24(5): 648-655. 
The majority of atypical antipsychotics showed antagonistic/partial agonistic effects on serotonergic receptors had increased efforts to investigate the role of serotonin in the pathophysiology of schizophrenia. Lysergic acid diethylamide, which has an agonistic effect on the serotonergic system, has been shown to cause psychosis-like behaviors but rarely produces other nuclear symptoms of schizophrenia [7]. It has also been indicated that a single dose of m-chlorophenylpiperazine, an agonist of serotonergic receptors, did not produce psychotic symptoms [8]. It has been demonstrated that there is an increase in the serotonin level and its metabolites in the basal ganglia and a decrease in serotonergic 5-HT2 receptor density in the prefrontal cortex of schizophrenia patients. Certain studies investigating the amount of serotonin and its major metabolite, 5-hydroxy indole acetic acid, have conflicting results in cerebrospinal fluid [9]. In the light of all findings, it is thought that the increase of serotonergic function in the subcortical region and the decrease of serotonergic activity in prefrontal regions may cause the pathophysiology of positive and negative symptoms, respectively.

Vortioxetine is one of the latest antidepressant drugs approved by the Food and Drug Administration (FDA) in September 2013. It has been known that vortioxetine's mechanism of action is different from other selective serotonin reuptake inhibitors (SSRI) since it modulates various serotonin receptors. It has been shown that vortioxetine blocks the serotonin reuptake transporter with a high affinity like SSRIs. Besides, it has been proved that vortioxetine shows agonistic effects on 5-HT1A and partial agonist on 5-HT1B receptors while it antagonizes the serotonergic 5-HT3 and 5-HT7 receptors [10]. All the information mentioned above considered together; it has been thought that vortioxetine might be a beneficial effect on schizophrenia because of its multimodal regulatory role in the serotonergic system in the brain. The limited number of studies examining the effect of vortioxetine on patients with schizophrenia indicated that vortioxetine supplementation to antipsychotic medicines (risperidone and clozapine) might produce extra benefits on cognitive or negative but not positive symptoms of disease [11,12].

Herein, we have first investigated the effects of alone administrated vortioxetine, not add on treatment, on schizophrenia-like behaviors in the acute ketamine model of schizophrenia in rats. We have performed novel object recognition (NOR), social interaction (SI), and prepulse inhibition (PPI) of acoustic startle response tests to evaluate positive, negative, and cognitive symptoms like behaviors in rats. The NOR task was used to test the visual recognition memory controlled by hippocampal-perirhinal cortical networks in rodents [13]. PPI test was also performed to evaluate sensorimotor gating function regulated by cortex, striatum, pallidum and pontine tegmentum neuronal networks, which is disrupted in certain neuropsychiatric disorders including schizophrenia and some of the cognitive disorders [14]. SI test was conducted to evaluate the social function, one of the unmet needs of schizophrenia. In this study, it was aimed to research whether vortioxetine improved the deficits of sensorimotor gating, visual recognition and social functions via versatile serotonergic modulatory effects of it in acute ketamine model of schizophrenia in rats.

\section{RESULTS}

\subsection{Vortioxetine decreased the avoiding behavior in SI test}

Our results showed that ketamine administration decreased the following $(\mathrm{p}<0.01)$ and sniffing $(\mathrm{p}<0.001)$ behaviors and increased the avoiding $(\mathrm{p}<0.05)$ behavior compared to the saline group. Vortioxetine alone administration did not alter the spent time for social behaviors in comparison with saline. Vortioxetine administration combined with ketamine significantly decreased $(\mathrm{p}<0.05)$ the avoiding behavior compared to ketamine. However, vortioxetine injected rats did not have altered following and sniffing behaviors than ketamine groups even though there were certain tendencies for an increase in related groups (Figure 1).

\subsection{Vortioxetine increased the discrimination index in the NOR task}

The ketamine administered rats had significantly lower $(\mathrm{p}<0.001)$ the discrimination index than salineinjected rats in the NOR task. However, the discrimination index of the vortioxetine alone group was not changed according to the saline group. Vortioxetine treatment after ketamine caused an increased $(\mathrm{p}<0.05)$ discrimination index compared to ketamine (Figure 2). 
A

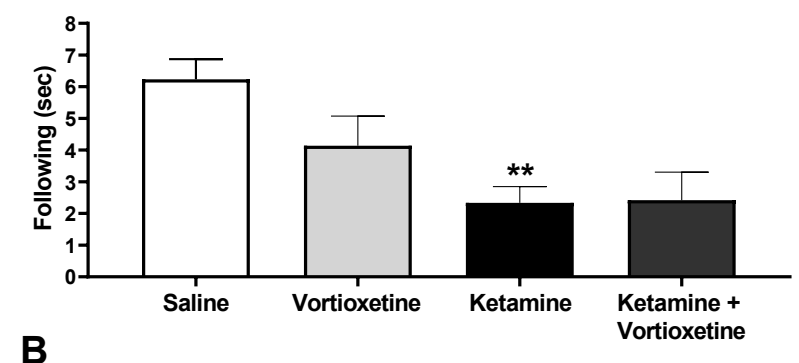

B

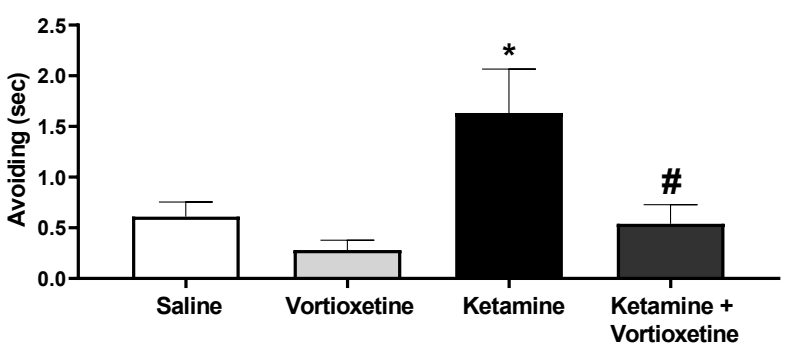

C

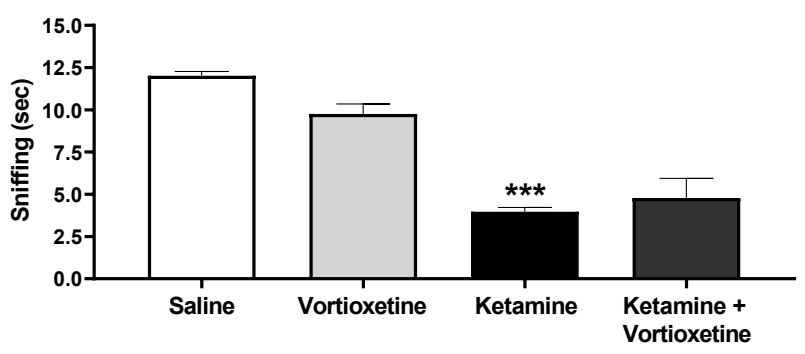

Figure 1. Following (A), avoiding (B), and sniffing (C) behaviors of rats in saline, vortioxetine, ketamine, and ketamine + vortioxetine groups. Statistical analyses were done with one way ANOVA followed by Dunnett's post hoc test. Data were presented as mean \pm SEM. ${ }^{*}: \mathrm{p}<0.05,{ }^{* *}: \mathrm{p}<0.01,{ }^{* * *}: \mathrm{p}<0.001$ compared to the saline group; \#: $\mathrm{p}<0.05$ compared to ketamine.

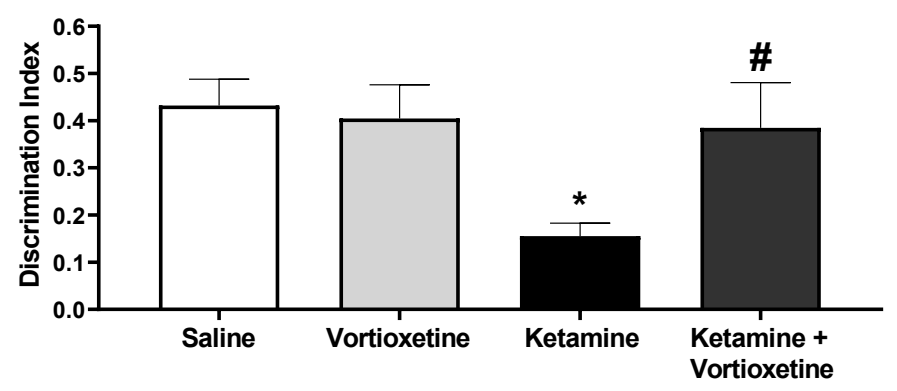

Figure 2. Discrimination indexes of saline, vortioxetine, ketamine, and ketamine + vortioxetine administered rats in NOR test. Statistical analyses were done with one way ANOVA followed by Dunnett's post hoc test. Data were presented as mean \pm SEM. *: $p<0.05$ compared to the saline; \#:p<0.05 compared to ketamine.

\subsection{Vortioxetine worsened the ketamine induced deficits in the PPI test}

The results indicated that ketamine administration decreased the prepulse inhibition (\%) compared to saline in $+4 \mathrm{~dB}(\mathrm{p}<0.001),+8 \mathrm{~dB}(\mathrm{p}<0.001)$, and $+16 \mathrm{~dB}(\mathrm{p}<0.001)$ prepulse intensities in the PPI test. Vortioxetine alone administration did not change the prepulse inhibition (\%) in none of the prepulse intensities in the PPI test. Ketamine+Vortioxetine group had significantly lower prepulse inhibition (\%) compared to ketamine group in $+4 \mathrm{~dB}(\mathrm{p}<0.05)+8 \mathrm{~dB}(\mathrm{p}<0.05)$ and $+16 \mathrm{~dB}(\mathrm{p}<0.05)$ prepulse intensities in PPI test (Figure $3)$. 


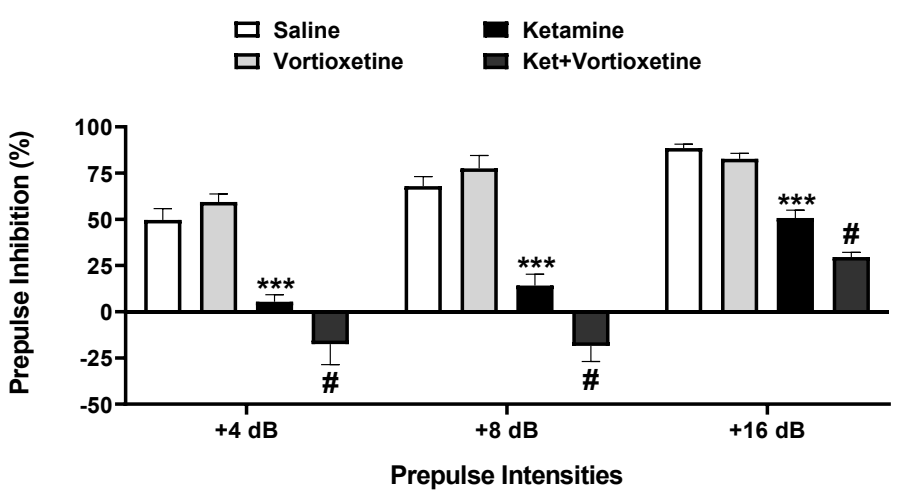

Figure 3. Prepulse inhibition (\%) of acoustic startle responses of saline, vortioxetine, ketamine, and ketamine + vortioxetine administered rats. Statistical analyses were done with two way ANOVA followed by Dunnett's post hoc test. Data were presented as mean $\pm S E M{ }^{* * *}: \mathrm{p}<0.001$ compared to saline; \#: $p<0.05$ compared to ketamine group.

In addition to the prepulse inhibition (\%), startle amplitude against pulse alone trials, and no stimulus trials were also calculated in the PPI test. Our results have shown that ketamine administration significantly decreased $(\mathrm{p}<0.001)$ the startle amplitude of pulse alone trials. At the same time, it markedly increased $(\mathrm{p}<0.001)$ the basal startle amplitude of no stimulus trials compared to saline in the PPI test. However, vortioxetine administration did not alter the startle amplitudes against pulse alone or no stimulus trials in the PPI test (Figure 4).

A

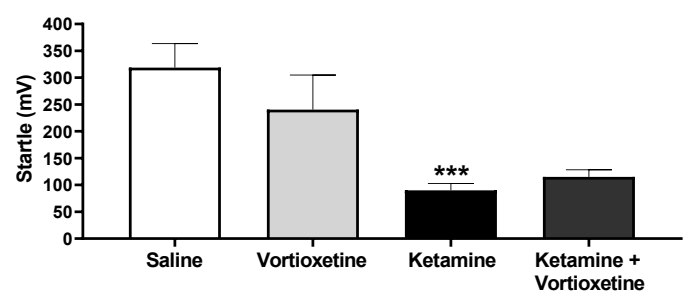

B

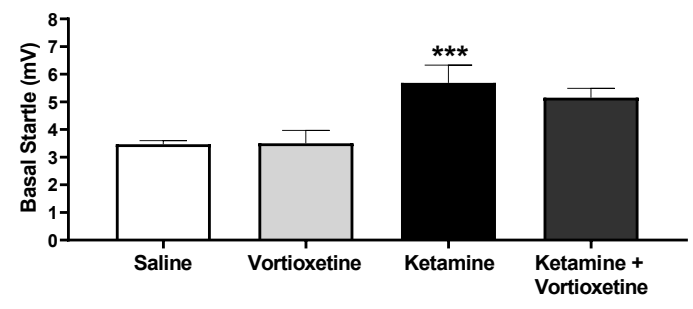

Figure 4. Startle amplitudes of pulse alone trials (A) and no stimulus (B) trials of saline, vortioxetine, ketamine, and ketamine + vortioxetine administered rats in PPI test. Statistical analyses were done with one way ANOVA followed by Dunnett's post hoc test. Data were presented as mean \pm SEM. ${ }^{* * *}: \mathrm{p}<0.05$ compared to the saline group.

\section{DISCUSSION}

In this study, we have evaluated the potential beneficial effects of vortioxetine, a new multimodal antidepressant, in the acute ketamine model of schizophrenia in rats. Our results showed that acute ketamine administration caused schizophrenia-like behaviors in rats. Ketamine administration disrupted cognitive performance, social behaviors, and sensorimotor gating mechanisms tested by rats' prepulse inhibition. Besides, vortioxetine alone administration did not alter the physiological functions of recognition memory, social behaviors, and sensorimotor gating in rats. However, vortioxetine treatment reversed the recognition memory and partially social deficits but not sensorimotor gating deficits in rats. Moreover, vortioxetine treatment deteriorated the ketamine-induced sensorimotor gating deficit in rats. These results suggest that vortioxetine treatment improved negative and cognitive symptoms like behaviors but not sensorimotor gating deficit in the acute ketamine model of schizophrenia in rats.

Social interaction is a valuable translational tool to evaluate social deficits, a component of negative symptoms of schizophrenia, in rodents. Sam's Dodd et al. (1999) showed that NMDA receptor antagonism caused social deficits in rats, and the model had a face and predictive validity in social interaction tests [15]. Different studies have also shown that acute ketamine administration disrupted social behaviors in rats [16]. To the best of our knowledge, there is only one study investigating the effect of vortioxetine on social behaviors 
in rodents. Witt et al. (2019) have indicated that vortioxetine treatment transiently increased the social sniffing behavior in mice models of autism spectrum disorders. In the same study, researchers have suggested that prefrontal serotonergic enhancement, especially SERT inhibition and 5-HT1B receptor partial agonism, played a role in the effect of vortioxetine in mice [17]. Besides, another study has demonstrated that a specific 5-HT-7 receptor antagonist (SB-269970) improved ketamine-induced social deficits in rats [16]. When the studies and pharmacological profiles of vortioxetine (SERT inhibition, 5-HT1B receptor partial agonism, and 5-HT-7 receptor antagonism) are considered together, our results indicate the social enhancer effect of vortioxetine was compatible with the previous studies. Our results demonstrated that vortioxetine might provide benefits for the social deficits of schizophrenia.

One of the primer initiatives aiming to treat cognitive deficits of schizophrenia, The Measurement and Treatment Research to Improve Cognition in Schizophrenia (MATRICS), advise the novel object recognition test as a translational paradigm evaluating visual recognition memory in rodents [18]. Previous reports showed that NMDA receptor antagonists, such as ketamine, deteriorated the visual recognition memory in rodents [5]. It has been suggested that a novel object recognition test is a well-validated task to evaluate cognitive dysfunction due to NMDA receptor antagonism in rats [19]. Several studies indicated the procognitive effects of vortioxetine in patients with depression or various rodent models [20-22]. Bruno et al. (2020) demonstrated the cognitive enhancer effect of vortioxetine in schizophrenic patients in a pilot study [11]. In a preclinical study, it has been reported that acute and subchronic administration of vortioxetine improved the cognitive deficits in a well-validated schizophrenia model, subchronic phencyclidine model rats [23]. Several hypotheses, such as serotonergic, glutamatergic, GABAergic, or histaminergic enrichments in the prefrontal cortex has been suggested for the precognitive effects of vortioxetine in previous studies [19-21]. Our findings have first shown vortioxetine's procognitive effects in the acute ketamine model of schizophrenia in rats.

The PPI of acoustic startle response is a translational tool evaluating sensorimotor gating function, which has been reported disrupted in schizophrenia [14]. Previous studies have demonstrated that ketamine administration disrupted the sensorimotor gating function in healthy volunteers and rodents $[4,24,25]$. The fact that the sensorimotor gating deficit induced by ketamine restored by antipsychotic drugs, and this literature reports made ketamine a valuable model that had the face and construct validities for the PPI test [26]. To the best of our knowledge, there was no study examining the effect of vortioxetine on the sensorimotor gating mechanism of the brain. Herein, we firstly showed that vortioxetine alone administration did not alter the sensorimotor gating function. However, vortioxetine deteriorated the ketamine-induced sensorimotor gating deficit in rats. It has been known that increased serotonergic activity in subcortical regions such as the limbic system, may be responsible for positive symptoms of schizophrenia [8]. When the multimodal serotonergic enhancer properties of vortioxetine and cortico-pallido-striato-thalamic control of sensorimotor gating are considered together, it has been thought that the synergistic disruptive effect of vortioxetine and ketamine on sensorimotor gating is consistent with the theoretical knowledge [10,14]. In this study, we first showed the aggravative effect of vortioxetine on sensorimotor gating deficit and related motor deficits examined with startle amplitude against the acoustic stimulus.

Our study has some improvable limitations though it firstly investigates the effect of vortioxetine on core symptoms of schizophrenia in the acute NMDA receptor antagonist model in rats. The ex vivo molecular and biochemical analyses for well-known and repeated parameters in schizophrenia-related brain regions could help understand the underlying mechanism of beneficial effects of vortioxetine in social and cognitive deficits in rats. It will also be precious to investigate the acute and chronic effects of vortioxetine in different rodent models, which well-mimicking the neurobiological findings of schizophrenia.

\section{CONCLUSION}

In this study, we have investigated the effects of vortioxetine on core symptoms of schizophrenia in the acute ketamine model of rats. Herein, we have reported that vortioxetine improved the social and cognitive deficits induced by ketamine in rats. However, we have first reported that vortioxetine may worsen the sensorimotor gating deficit of schizophrenia. In light of all the results of our study, we have suggested that the effect of vortioxetine is worth investigating for negative and cognitive symptoms of schizophrenia, which are the unmet needs of the disease. However, the deteriorative effect of vortioxetine on sensorimotor gating needs to be confirmed with further studies. It will be valuable to focus on the underlying mechanism of potential beneficial effects of vortioxetine for a better understanding of its effects and produce further treatment strategies for the treatment of schizophrenia. 


\section{MATERIALS AND METHODS}

\subsection{Animals and housing}

All experiments were conducted in this study were approved by the Erciyes University Animal Research Ethics Committee (Approval number: 20099). All animals were brought to the laboratory one week before behavioral tests. Standard care procedures, including constant temperature $\left(22 \pm 1^{\circ} \mathrm{C}\right), 12 / 12$ light and dark cycle, no restriction for feeding and water were applied in our experiments. Wistar albino rats (16 weeks) were grouped as saline, vortioxetine $(10 \mathrm{mg} / \mathrm{kg})$, ketamine $(10 \mathrm{mg} / \mathrm{kg}$ for PPI, $15 \mathrm{mg} / \mathrm{kg}$ for other tests $)$ and ketamine + vortioxetine $(10 \mathrm{mg} / \mathrm{kg})$. The eight rats were used in each group.

\subsection{Drugs and experimental design}

Ketamine (Pfizer Pharmaceuticals, USA) was diluted with saline. Vortioxetine (Lundbeck, Denmark) was dissolved in saline. All injections were applied at a volume of $0.1 \mathrm{ml} / 100 \mathrm{~g}$. Ketamine $(10 \mathrm{mg} / \mathrm{kg})$ was subcutaneously injected to rats $15 \mathrm{~min}$ before the prepulse inhibition of the acoustic startle response (PPI) test. Ketamine $(15 \mathrm{mg} / \mathrm{kg}$ ) was injected $45 \mathrm{~min}$ before the tests for novel object recognition (NOR), social interaction (SI) tests. Different dose and application time were determined according to our and other previous studies $[5,14,24,27]$. Almost all previous studies considered that subcutaneously injection 15 min prior to the test was optimal protocol for PPI test $[14,24]$. In addition to this, our previous study indicated that $10 \mathrm{mg} / \mathrm{kg}$ ketamine injection is sufficient for a disruption in sensorimotor gating in rats [5]. Ketamine $(15 \mathrm{mg} / \mathrm{kg})$ is preffered to minimal effect of ketamine on locomotor activity in rats. Vortioxetine $(10 \mathrm{mg} / \mathrm{kg}) \mathrm{was}$ intraperitoneally administered $30 \mathrm{~min}$ before all behavioral tests. A five-day washout period was used between behavioral tests.

\subsection{Behavioral tests}

\subsubsection{Social interaction (SI) test}

SI test was conducted in a black plexiglass open field arena $(50 \times 50 \times 40 \mathrm{~cm})$ at dimly-lit conditions. In this test, two unfamiliar rats allowed to interact with each other for 10 minutes. One of the rats was colored with temporary and non-odor ink for easy discrimination by the researcher. All experiments were recorded with a video camera placed on the top of the apparatus. Social behaviors were scored by a blind researcher for each rat. The time spent for the sniffing and following behaviors was measured as an indicator of socialization, and spending time for avoiding was considered an indicator of social withdrawal. The test apparatus was cleaned with non-odor disinfectant between each test [27].

\subsubsection{Novel object recognition (NOR) test}

The test was conducted in a black plexiglass box $(50 \times 50 \times 40 \mathrm{~cm})$ in a dimly lit condition. The NOR test consisted of two different periods performed on two consecutive days. The first day, habituation, all animals in the same groups allowed to move freely in the test apparatus for 60 minutes. No object was used in the habituation period. The second day, test day consisted of two different 3-minute trials with a 1-hour intertrial interval. In trial 1 (Familiarization, T1), two identical objects were placed on the opposite corners of the chamber (10 cm from each wall). The rats were permitted to move freely and explore this chamber for 3 min. Then, one of the identical objects (A for each) was exchanged with a novel one (B) in a 1-hour interval. After that, the same rat tested on the second trial (Retention, T2). In the retention period, the rats also allowed to move in the test chamber freely. All experiments were recorded by a camera, and exploration times were scored by a blind researcher for every object in T2. The test apparatus was cleaned by non-odor disinfectant between each test. The discrimination index (DI) was calculated with the following formula: DI = (EB $\mathrm{EA}) /(\mathrm{EB}+\mathrm{EA})$ for each animal [13].

\subsubsection{Prepulse inhibition (PPI) of acoustic startle response test}

The SR-LAB systems (San Diego Instruments, San Diego, USA) were used to measure PPI of acoustic startle responses in rats. This system consists of two soundproof test chambers $(39 \times 38 \times 58 \mathrm{~cm})$, plexiglass cylinder restrainer in chambers, piezoelectric sensors for measuring startle, and loudspeakers for producing acoustic stimulus. The test protocol was described in previous reports [24]. Briefly, the PPI test was performed on two consecutive days, including habituation and test days. In habituation day, the rats have put the chambers for five minutes. In this period, the rats exposed to continuous $70 \mathrm{~dB}$ background noise for acclimation to test environment and five severe $(120 \mathrm{~dB})$ startle stimuli to test hearing and motor functions. In 
test day, the rats were exposed to background noise for $5 \mathrm{~min}$ and three blocks of trials. Block 1 included five presentations of a $40 \mathrm{~ms} 120 \mathrm{~dB}$ pulse trial. Block 2 consisted of 50 pseudo-random trials with changing intertrial intervals with 15 -sec average which included eight presentations of each prepulses+pulse trials (20 $\mathrm{ms}+4(74) \mathrm{dB},+8(78) \mathrm{dB}$, and $+16(86) \mathrm{dB}$ prepulse $100 \mathrm{~ms}$ before the $40 \mathrm{~ms} 120 \mathrm{~dB}$ pulse), ten presentations of pulse alone $(120 \mathrm{~dB})$ trials, and ten presentations of no stimulus (only background noise) trials. Block 3 includes the five presentations of pulse trials. The percent prepulse inhibition was calculated from block 2 , whereas block 1 and block 3 were used to evaluate getting used to the startle stimulus. The following formula was used to calculate the percent prepulse inhibition: \%PPI= $100-(\mathrm{PP}) /(\mathrm{P}) \times 100$. "PP" and "P" means the startle response after the presentation of prepulse+pulse stimulus and pulse stimulus, respectively. Startle response to pulse-alone trials and basal activity in no stimulus trials were also evaluated as additional parameters in this study.

\subsection{Statistical analysis}

The statistical analyses were performed in Graph Pad Prism 8 software. One way analysis of variance (ANOVA) was used for social behaviors in SI, discrimination indexes in NOR and the startle amplitudes in PPI tests. The two way ANOVA test was performed to evaluate percent PPI in each prepulse intensity. Dunnett's test was used as a post hoc test in all analyses. Data were presented as mean \pm standard error of the mean (SEM), and $\mathrm{p}<0.05$ was considered a value of statistical significance.

Acknowledgements: We would like to thank Prof. Dr. Asuman Golgeli for her support to Prepulse Inhibition of Acoustic Startle Response Test.

Author contributions: Concept - G.U.; Design - G.U.; Resources - G.U.; Materials - G.U., M.T.; Data Collection and/or Processing - G.U., M.T.; Analysis and/or Interpretation -G.U.; Literature Search - G.U.; Writing - G.U.; M.T.; Critical Reviews - G.U., M.T.

Conflict of interest statement: The authors declared no conflict of interest.

Ethics committee approval: All experiments were conducted in this study were approved by the Erciyes University Animal Research Ethics Committee with the approval number of 20099 on July 12020.

\section{REFERENCES}

[1] van Os J, Kapur S. Schizophrenia. Lancet. 2009; 374(9690): 635-645. [CrossRef]

[2] Naber D, Lambert M. The CATIE and CUtLASS studies in schizophrenia: results and implications for clinicians. CNS Drugs. 2009; 23(8): 649-659. [CrossRef]

[3] Stahl SM. Beyond the dopamine hypothesis of schizophrenia to three neural networks of psychosis: dopamine, serotonin, and glutamate. CNS Spectr. 2018; 23(3): 187-191. [CrossRef]

[4] Boeijinga PH, Soufflet L, Santoro F, Luthringer R. Ketamine effects on CNS responses assessed with MEG/EEG in a passive auditory sensory-gating paradigm: an attempt for modelling some symptoms of psychosis in man [published correction appears in J Psychopharmacol. 2007; 21(8): 900]. J Psychopharmacol. 2007; 21(3): 321-337. [CrossRef]

[5] Unal G, Aricioglu F. Famotidine Improved Schizophrenia-Like Behaviors in Acute Ketamine Model of Schizophrenia in Rats. Psychiatry and Behavioral Sciences. 2020; 10(2): 45-54. [CrossRef]

[6] Cadinu D, Grayson B, Podda G, Harte MK, Doostdar N, Neill JC. NMDA receptor antagonist rodent models for cognition in schizophrenia and identification of novel drug treatments, an update. Neuropharmacology. 2018; 142: 41-62. [CrossRef]

[7] De Gregorio D, Comai S, Posa L, Gobbi G. d-Lysergic Acid Diethylamide (LSD) as a Model of Psychosis: Mechanism of Action and Pharmacology. Int J Mol Sci. 2016; 17(11): 1953. [CrossRef]

[8] Steeds H, Carhart-Harris RL, Stone JM. Drug models of schizophrenia. Ther Adv Psychopharmacol. 2015; 5(1): 43-58. [CrossRef]

[9] Keshavan MS, Tandon R, Boutros NN, Nasrallah HA. Schizophrenia, "just the facts": what we know in 2008 Part 3: neurobiology. Schizophr Res. 2008; 106(2-3): 89-107. [CrossRef]

[10] D'Agostino A, English CD, Rey JA. Vortioxetine (brintellix): a new serotonergic antidepressant. P T. 2015; 40(1): 3640. 
[11] Bruno A, Zoccali RA, Troili GM, et al. Vortioxetine on Cognition in Schizophrenia: A Pilot Study. J Clin Psychopharmacol. 2020; 40(4): 381-385. [CrossRef]

[12] Moazen-Zadeh E, Bayanati S, Ziafat K, Rezaei F, Mesgarpour B, Akhondzadeh S. Vortioxetine as adjunctive therapy to risperidone for treatment of patients with chronic schizophrenia: A randomised, double-blind, placebo-controlled clinical trial. J Psychopharmacol. 2020; 34(5): 506-513. [CrossRef]

[13] Antunes M, Biala G. The novel object recognition memory: neurobiology, test procedure, and its modifications. Cogn Process. 2012; 13(2): 93-110. [CrossRef]

[14] Swerdlow NR, Braff DL, Geyer MA. Sensorimotor gating of the startle reflex: what we said 25 years ago, what has happened since then, and what comes next. J Psychopharmacol. 2016; 30(11): 1072-1081. [CrossRef]

[15] Sams-Dodd, F. Phencyclidine in the social interaction test: an animal model of schizophrenia with face and predictive validity. Rev Neurosci. 1999; 10: 59-90. [CrossRef]

[16] Hołuj M, Popik P, Nikiforuk A. Improvement of ketamine-induced social withdrawal in rats: the role of 5-HT7 receptors. Behav Pharmacol. 2015; 26: 766-775. [CrossRef]

[17] Witt NA, Lee B, Ghent K, et al. Vortioxetine Reduces Marble Burying but Only Transiently Enhances Social Interaction Preference in Adult Male BTBR T+Itpr3tf/J Mice. ACS Chem Neurosci. 2019; 10(10): 4319-4327. [CrossRef]

[18] Redrobe JP, Bull S, Plath N. Translational Aspects of the Novel Object Recognition Task in Rats Abstinent Following Sub-Chronic Treatment with Phencyclidine (PCP): Effects of Modafinil and Relevance to Cognitive Deficits in Schizophrenia. Front Psychiatry. 2010; 1: 146. [CrossRef]

[19] Neill JC, Barnes S, Cook S, et al. Animal models of cognitive dysfunction and negative symptoms of schizophrenia: focus on NMDA receptor antagonism. Pharmacol Ther. 2010; 128(3): 419-432. [CrossRef]

[20] Mahableshwarkar AR, Zajecka J, Jacobson W, Chen Y, Keefe RS. A Randomized, Placebo-Controlled, ActiveReference, Double-Blind, Flexible-Dose Study of the Efficacy of Vortioxetine on Cognitive Function in Major Depressive Disorder [published correction appears in Neuropsychopharmacology. 2016 Nov;41(12):2961]. Neuropsychopharmacology. 2015; 40(8): 2025-2037. [CrossRef]

[21] Bétry C, Etiévant A, Pehrson A, Sánchez C, Haddjeri N. Effect of the multimodal acting antidepressant vortioxetine on rat hippocampal plasticity and recognition memory. Prog Neuropsychopharmacol Biol Psychiatry. 2015; 58: 3846. [CrossRef]

[22] Smagin GN, Song D, Budac DP, et al. Histamine may contribute to vortioxetine's procognitive effects; possibly through an orexigenic mechanism. Prog Neuropsychopharmacol Biol Psychiatry. 2016; 68: 25-30. [CrossRef]

[23] Pehrson AL, Pedersen CS, Tølbøl KS, Sanchez C. Vortioxetine Treatment Reverses Subchronic PCP TreatmentInduced Cognitive Impairments: A Potential Role for Serotonin Receptor-Mediated Regulation of GABA Neurotransmission. Front Pharmacol. 2018; 9: 162. [CrossRef]

[24] Mansbach RS, Geyer MA, Braff DL. Dopaminergic stimulation disrupts sensorimotor gating in the rat. Psychopharmacology (Berl). 1988; 94(4): 507-514. [CrossRef]

[25] Martinez ZA, Ellison GD, Geyer MA, Swerdlow NR. Effects of sustained phencyclidine exposure on sensorimotor gating of startle in rats. Neuropsychopharmacology. 1999; 21(1): 28-39. [CrossRef]

[26] Swerdlow NR, Bakshi V, Waikar M, Taaid N, Geyer MA. Seroquel, clozapine and chlorpromazine restore sensorimotor gating in ketamine-treated rats. Psychopharmacology (Berl). 1998; 140(1): 75-80. [CrossRef]

[27] Nikiforuk A, Hołuj M, Kos T, Popik P. The effects of a 5-HT5A receptor antagonist in a ketamine-based rat model of cognitive dysfunction and the negative symptoms of schizophrenia. Neuropharmacology. 2016; 105: 351-360. [CrossRef] 